\title{
Cable force estimation of cables with small sag considering inclination angle effect
}

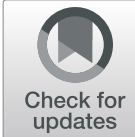

Wen-Yu He ${ }^{1,2}$, Fan-Cheng Meng ${ }^{1,2}$ and Wei-Xin Ren ${ }^{3 *}$

\footnotetext{
* Correspondence: renwx@szu.edu.

${ }^{3}$ College of Civil and Transportation Engineering, Shenzhen University, Shenzhen 518061, Guangdong Province, China

Full list of author information is available at the end of the article
}

\begin{abstract}
Cable force estimation is essential for security assessment of cable-stayed bridges. Cable force estimation methods based on the relationship between cable force and frequency have been extensively studied and used during both construction phase and service phase. However, the effect induced by inclination angle of the cable is not included in the establishment of frequency-cable force relationship as horizontal cable model is normally employed. This study aims to investigate the influence of the inclination angle on vibration based cable force estimation and provide practical formulas accordingly. Firstly numerical examples of fixed-fixed and hinged-hinged cables are simulated to illustrate the necessity of considering the inclination angle effect on the modal parameters and cable force estimation for inclined cables with small sag. Then practical formulas considering the inclination angle effect to estimate the cable force of fixed-fixed and hinged-hinged cables via the fundamental frequency are established accordingly. For the inclined cables with unknown boundary conditions, the coefficients reflecting boundary condition are predicted via the practical formulas for fixed-fixed and hinged-hinged cables. And the cable force considering the influence of inclination angle and unknown boundary conditions is obtained by iteration method. Finally, numerical examples are presented to demonstrate the effectiveness of the proposed method.
\end{abstract}

Keywords: Cable force estimation, Inclination angle, Boundary condition, Frequency

\section{Introduction}

Cable-stayed bridges are widely used due to their reasonable structural force, beautiful shape, mature construction methods and large span (Zhang et al. 2020). The cable is the main stressed member of the cable-stayed bridge, and its health status affects the safety of the bridge. Cable damages induced by corrosion, fatigue and other reasons, may lead to bridge collapse and other malignant accidents. Therefore cable force has become one of the most important indexes for cable-stayed bridge condition assessment (Ren and Hu 2009; Zhao et al. 2020; Tian et al. 2021).

Generally the cable force estimation methods can be divided into direct methods and indirect methods (Ren et al. 2005; Kim et al. 2007). The direct method refers to the direct measurement of the cable force, including the manometer method and the pressure sensor method. On the other hand, indirect method estimates the cable force

(c) The Author(s). 2021 Open Access This article is licensed under a Creative Commons Attribution 4.0 International License, which permits use, sharing, adaptation, distribution and reproduction in any medium or format, as long as you give appropriate credit to the original author(s) and the source, provide a link to the Creative Commons licence, and indicate if changes were made. The images or other third party material in this article are included in the article's Creative Commons licence, unless indicated otherwise in a credit line to the material. If material is not included in the article's Creative Commons licence and your intended use is not permitted by statutory regulation or exceeds the permitted use, you will need to obtain permission directly from the copyright holder. To view a copy of this licence, visit http://creativecommons.org/licenses/by/4.0/. 
by measuring indirect physical quantities, such as magnetic flux method and vibrationbased method. Vibration based method is the most widely used method for its high precision, convenient implementation and low cost. The main principle is that the cable force can be estimated by the measured frequencies according to the specific relationship between the cable force and frequency (Cunha et al. 2001). During the process of establishing the relationship between the cable force and frequency, various factors, such as bending stiffness, sag, boundary conditions, and temperature are often simplified or ignored, which would lead to non-negligible errors in the estimated cable force (Dan et al. 2014; Chen et al. 2018).

For slender cables, the bending stiffness of the cable plays a very weak role in vibration, and its vibration basically follows the string vibration theory (Ceballos and Prato 2008). However, the bending stiffness of the short and thick cables cannot be ignored, and their vibrations are closer to the Euler Bernoulli beam (Fang and Wang 2012). If the string vibration theory was still adopted, the calculated cable force would be larger.

Three hinds of boundary conditions specific as hinged-hinged, fixed-fixed, and hinged-fixed are normally adopted in the vibration based cable force estimation. The beam model with hinged-hinged boundary conditions has definite analytical solutions, thus the corresponding cable force can be solved directly by using the cable parameters. Since there are no analytical solutions for beam models with fixed-fixed and hingedfixed boundary conditions, numerical solutions are normally required (Huang et al. 2014). The concept of effective calculation length was introduced to transform the fixed boundary into the hinged boundary, then the solution for hinged-hinged boundary condition could be adopted (Yan et al. 2015). Actually the boundary condition of cable is elastic embedded boundary between hinged and fixed instead of ideal hinged or fixed (Huang et al. 2015). On the one hand, the anchorage form of the cable end is complex with various degrees of elastic embedded. Interpolation method can be used to get the coefficients that reflecting the degrees of elastic embedded boundary and the cable force can be further obtained iteratively. On the other hand, in order to inhibit the large vibration and fatigue failure of the cable, dampers are usually installed at the connection location between the cable and bridge deck, which result in more complex boundary conditions and vibration performance (Yan et al. 2019; Shi and Zhu 2018).

The cable is generally arranged as a catenary due to the dead weight. The cable sag affects both the natural frequency and cable force estimation. Studies reveal that sag has a great influence on the fundamental frequency, but little influence on the even order frequencies (Zui 1996). When the cable length is long and the cable force is small, the sag effect must be considered in cable force estimation. In this case, the even order frequency can be used to improve the accuracy (Zui 1996).

The influence of temperature on cable force is mainly caused by the daily temperature range. As the thermal conductivity coefficients vary depending on the materials, the same temperature change will induce a large temperature difference among cables, beams and towers, which in turn generate a large additional internal force (Zhao et al. 2017; Feng et al. 2019; Ma et al. 2021). The cable forces of cable-stayed bridges are usually designed according to the closure temperature, so the test temperature should be as consistent as possible with the closure temperature to minimize the temperature influence. 
Though great efforts have been paid on various factors as mentioned above, the inclination angle effect attracts little attention. Most of the existing methods take horizontal cable model to establish the relationship between cable force and frequency, without considering the influence of cable inclination. When solving the differential equation of cable vibration, the cable force is linearly distributed along the length due to its self-weight. However, the cable force is deemed as constant in the existing horizontal cable model, which will inevitably generate errors in the solution. Besides, inclination angle will give rise to the redistribute the dead weight of the cable and further affect the cable sag. Thus the accuracy of the modal parameters and cable force estimation would be reduced. In view of this, this study will investigate the influence of inclination angle on cable force estimation for cables with small sag, and provide practical cable force estimation formulas for engineering practice accordingly. Numerical studies will be conducted to demonstrate the effectiveness of the proposed practical formulas.

\section{Basic theory}

An inclined cable model considering the bending stiffness, inclination angle, sag, external force and boundary conditions is shown in Fig. 1. The in-plane motion equation $(y$ direction) of the cable can be expressed as (Ma 2017):

$$
\frac{\partial}{\partial s}\left[(T+\tau) \frac{\partial z}{\partial s}\right]-\frac{\partial^{2}(E I \kappa)}{\partial s^{2}}+m g \cos \theta=m \frac{\partial^{2} v}{\partial t^{2}}
$$

where $E I$ denotes the bending stiffness of the cable, $m(x)$ denotes the mass per unit length, $T$ and $\tau(x, t)$ denote the cable force and force increment caused by the free vibration, respectively.

The sum of the displacement in the $y$ direction is

$$
z(x, t)=\mathrm{y}(x)+v(x, t)
$$

where $y(x)$ and $v(x, t)$ denote the static deflection caused by the dead weight of the cable and the deflection caused by the vibration in the $y$ direction, respectively. $\mu(x)$ denotes the deflection in the $x$ direction, $d s=\left(d x^{2}+d y^{2}\right)^{1 / 2}$ denotes the infinitesimal length, $\theta$ denotes the inclination angle, and $\kappa$ denotes the bending curvature.

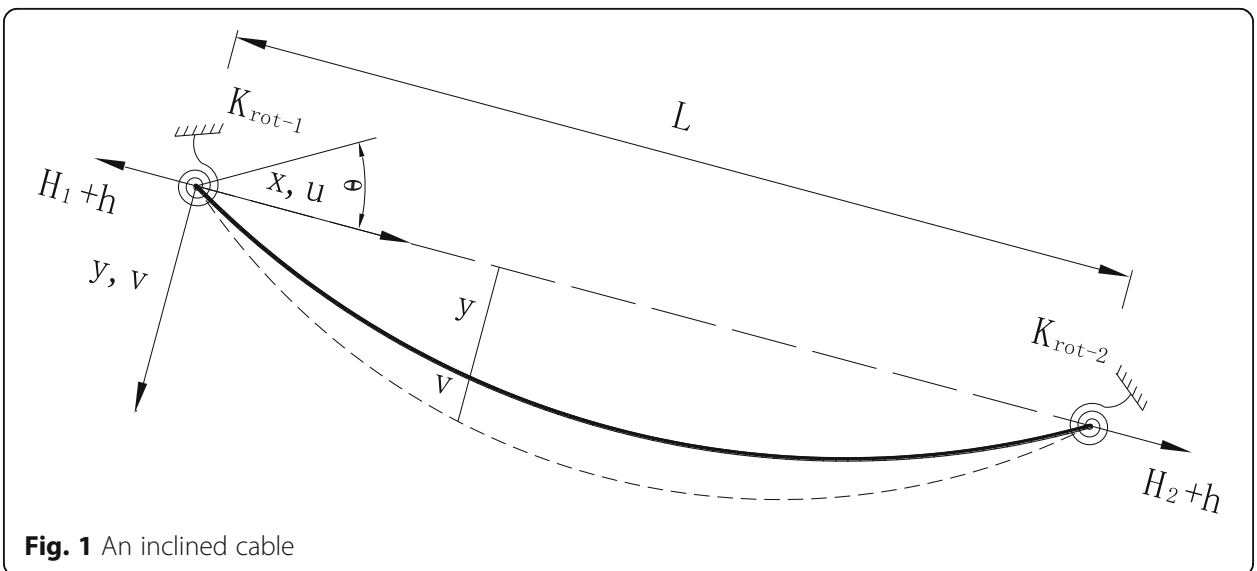


When the cable damping and axial component of the motion equation are not considered, the $d s=\left(d x^{2}+d y^{2}\right)^{1 / 2}$ can be replaced by $d x$ and Eq.(1) can be simplified as

$$
\frac{\partial}{\partial x}\left[(H+h) \frac{\partial z}{\partial x}\right]-\frac{\partial^{2}\left(E I z^{\prime \prime}\right)}{\partial x^{2}}+m g \cos \theta=m \frac{\partial^{2} v}{\partial t^{2}}
$$

where the first term is the cable force component in the $y$ direction, the second term is the second derivative of the bending moment, the third term is the dead weight component of the cable in the $x$ direction, and the fourth term is the inertia term. $H(x)$ and $h(t)$ are the cable force component in the $x$ direction and the additional component caused by vibration, respectively. The following relationship can be found

$$
T=H \cdot d s / d x
$$

When the sag-span ratio is less than $1 / 8$, the $H$ can be deemed as $T$ (Ma 2017). In the following description, the cable force is represented by $T$ for simplicity. By ignoring the second order term, Eq.(3) can be expressed as

$$
E I \frac{\partial^{4} v(x, t)}{\partial x^{4}}-T(x) \frac{\partial^{2} v(x, t)}{\partial x^{2}}-T^{\prime}(x) \frac{\partial v(x, t)}{\partial x}-h(t) \frac{d^{2} y}{d x^{2}}+m \frac{\partial^{2} v}{\partial t^{2}}=0
$$

Assuming the solution of Eq.(5) has the following form

$$
v(x, t)=\phi(x) \cdot q(t)
$$

where $\phi(x)$ and $q(t)$ are the shape function and generalized coordinate, respectively.

The deformation compatibility equation of the cable can be established according to the geometrical and elastic relations between stress and strain (Ma 2017)

$$
\frac{h(d s / d x)^{3}}{E A}=\frac{\partial \mu}{\partial x}+\frac{d y}{d x} \frac{\partial v}{\partial x}
$$

Combine Eq.(6) and Eq.(7), the motion equation of the cable is obtained as follows

$$
E I \frac{d^{4} \phi}{d x^{4}}-T \frac{d^{2} \phi}{d x^{2}}-T^{\prime} \frac{d \phi}{d x}+\frac{\int_{0}^{L} \frac{d^{2} y}{d x^{2}} \phi d x}{\int_{0}^{L} \frac{(d s / d x)^{3}}{E A} d x} \frac{d^{2} y}{d x^{2}}+m \omega^{2} \phi=0
$$

As the cable force $T$ varies along the chord line due to the influence of the inclined angle, Eq.(8) is a four-order differential equations with variable coefficients. In this paper, the finite difference method is used to solve this equation (Mehrabi and Tabatabai 1998). The cable is divided into $N$ segments equally with length of $l$ in the $x$ direction (Fig. 2).

The difference scheme of the function $\phi(x)$ is

$$
\begin{aligned}
& \frac{d \phi}{d x}=\frac{\phi_{i+1}-\phi_{i-1}}{2 l} \\
& \frac{d^{2} \phi}{d x^{2}}=\frac{\phi_{i+1}-2 \phi_{i}+\phi_{i-1}}{l^{2}} \\
& \frac{d^{3} \phi}{d x^{3}}=\frac{\phi_{i+2}-2 \phi_{i+1}+2 \phi_{i-1}-\phi_{i-2}}{2 l^{3}}
\end{aligned}
$$




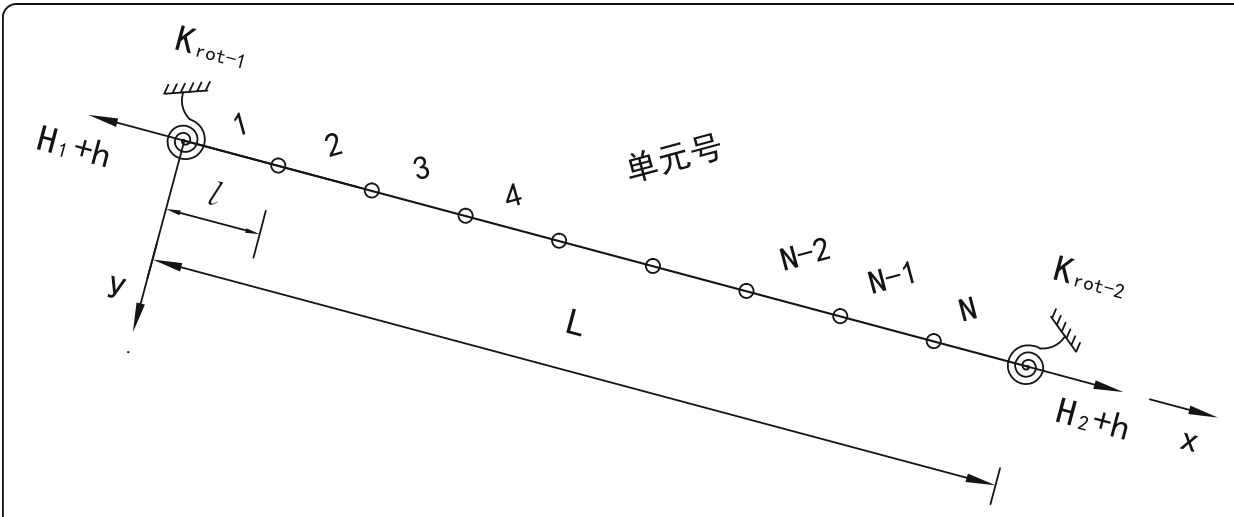

Fig. 2 Discrete cable model

$$
\frac{d^{4} \phi}{d x^{4}}=\frac{\phi_{i+2}-4 \phi_{i+1}+6 \phi_{i}-4 \phi_{i-1}+\phi_{i-2}}{l^{4}}
$$

Then the matrix form of the discrete equation is obtained for Eq.(8)

$$
\boldsymbol{K} \boldsymbol{w}=\boldsymbol{M} \omega^{2} \boldsymbol{w}
$$

where $\boldsymbol{K}=\boldsymbol{K}_{\boldsymbol{1}}+\boldsymbol{K}_{\mathbf{2}}, \boldsymbol{w}^{T}=\left\{w_{1}, w_{2}, \ldots, w_{k}\right\}$

$$
\mathbf{K}_{1}\left[\begin{array}{cccccc}
Q & U & W & & & \\
D & S & U & W & & \\
V & D & S & U & - & \\
& V & - & - & - & W \\
& & - & - & S & U \\
& & & V & D & G
\end{array}\right]_{k x k}
$$

where.

$$
\begin{aligned}
& S=\frac{1}{l^{4}}\left(-2 E I_{i+1}+10 E I_{i}-2 E I_{i-1}\right)+\frac{2 T_{i}}{l^{2}}, D=\frac{1}{l^{4}}\left(2 E I_{i+1}-6 E I_{i}\right)-\frac{T_{i}}{l^{2}}+\frac{T_{i+1}-T_{i-1}}{4 l^{2}} \\
& U=\frac{1}{l^{4}}\left(-6 E I_{i}+2 E I_{i-1}\right)-\frac{T_{i}}{l^{2}}-\frac{T_{i+1}-T_{i-1}}{4 l^{2}}, \\
& V=-\frac{1}{2 l^{4}}\left(E I_{i+1}-2 E I_{i}-E I_{i-1}\right), W=\frac{1}{2 l^{4}}\left(E I_{i+1}+2 E I_{i}-E I_{i-1}\right) \\
& Q=S+\frac{K_{\text {rot }-1} l-2 E I_{0}}{K_{\text {rot }-1} l+2 E I_{0}} V(i=1), G=S+\frac{K_{\text {rot }-2} l-2 E I_{k+1}}{K_{\text {rot }-2} l+2 E I_{k+1}} W(i=k)
\end{aligned}
$$

Each row in the matrix $\left(\boldsymbol{K}_{1}\right)$ corresponds to one node of the discrete cable, and $k$ denotes the node number of the cable except two ends. $E I_{0}$ and $E I_{k+1}$ are the bending stiffness of the two ends, respectively. $K_{r o t-1}$ and $K_{r o t-2}$ are the rotational stiffness of the two ends, respectively.

Due to the effect of the inclination, the cable forces are linearly distributed along the $x$ direction (Ma 2017). The cable forces at the top end and bottom end are:

$$
\begin{aligned}
& T_{0}=T_{M}+0.5 m g L \sin \theta \\
& T_{k+1}=T_{M}-0.5 m g L \sin \theta
\end{aligned}
$$

where $T_{M}$ is the average of the cable forces at both ends. The cable force at any node $(i)$ is

$$
T_{i}=T_{M}+m g \sin \theta(0.5 L-i)
$$

The nonlinear stiffness matrix $\boldsymbol{K}_{2}$ is 


$$
\mathbf{K}_{2}=\mathbf{r s}^{T}
$$

where.

$$
\begin{aligned}
& \mathbf{r}^{T}=\left\{r_{1}, r_{2}, \ldots, r_{k}\right\}, \mathbf{s}^{T}=\left\{s_{1}, s_{2}, \ldots, s_{k}\right\} \\
& r_{i}=\frac{s_{i}}{\sum_{1}^{k} t_{i}^{3} / E A_{i}}, s_{i}=\frac{y_{i+1}-2 y_{i}+y_{i-1}}{l^{2}}, t_{i}=\sqrt{1+\left(\frac{y_{i+1}-y_{i-1}}{2 l}\right)^{2}}
\end{aligned}
$$

The deflection curve of the cable is not a parabola as normally assumed when the influence of the inclination angle is considered. Thus it is calculated using the finite difference method according to the static equilibrium differential equation of the cable. The static equilibrium differential equation of a stay cable is:

$$
E I \frac{d^{4} y}{d x^{4}}-T \frac{d^{2} y}{d x^{2}}-T^{\prime} \frac{d y}{d x}=m g \cos \theta
$$

Eq. (15) is then discretized and the corresponding matrix form is

$$
\mathbf{K}_{1} \mathbf{Y}=\boldsymbol{m} g \cos \theta
$$

where $\boldsymbol{Y}=\left\{y_{1}, y_{2}, \ldots, y_{k}\right\}, y_{i}$ denotes the static deflection at the $i^{\text {th }}$ node, $\boldsymbol{m}=\left\{m_{1}, m_{2}, \ldots, m_{k}\right\}$.

$$
\mathbf{M}=\left(\begin{array}{ccc}
m_{1} & \cdots & 0 \\
\vdots & \ddots & \vdots \\
0 & \cdots & m_{k}
\end{array}\right)
$$

It should be noted that the stiffness matrix $K$ is asymmetric due to the effects of inclination angle.

\section{Influence of the inclination angle on the cable force estimation}

\subsection{Iterative method for cable force estimation}

As the characteristic frequency equation (Eq. 10) is a transcendental equation, there is no explicit formula for the cable force. The iterative method based on frequency sensitivity can be used to estimate cable force (Kim et al. 2007; Ma 2017). As this study mainly focus on inclination angle effect, other parameters such as bending stiffness and axial stiffness, are assumed to be constant in this section. Then Eq.(10) can be transformed into

$$
[\mathbf{K}(T)-\zeta(T) \mathbf{M}(T)] \mathbf{w}(T)=0
$$

where $\zeta(T)=\omega^{2}(T), \zeta$ and $\boldsymbol{w}(T)$ are the eigenvalue and corresponding eigenvector of Eq.(18), respectively. Eq.(18) can be further translated into

$$
\left[\mathbf{K}(T)-\zeta_{1} \mathbf{M}\right] \frac{d \mathbf{w}_{1}(T)}{d T}=\left[\zeta_{1} \frac{d \boldsymbol{M}}{d T}-\frac{d \boldsymbol{K}(T)}{d T}\right] \mathbf{w}_{1}(T)+\mathbf{M} \mathbf{w}_{1}(T) \frac{d \zeta_{1}(T)}{d T}
$$

The derivative of the frequency to the cable force is

$$
\frac{d \zeta_{1}(T)}{d T}=\left[\mathbf{w}_{1}^{T} \mathbf{M} \mathbf{w}_{1}\right]^{-1} \mathbf{w}_{1}^{T}\left[\frac{d \mathbf{K}(T)}{d T}-\zeta_{1} \frac{d \mathbf{M}}{d T}\right] \mathbf{w}_{1}
$$

The derivative of the matrix $\boldsymbol{K}_{\boldsymbol{I}}$ can be easily obtained via Eq.(11). The derivative of the matrix $K_{2}$ is 


$$
\frac{d \boldsymbol{K}_{2}(i, j)}{d T}=\frac{\partial\left(\boldsymbol{r}_{i} \boldsymbol{s}_{j}\right)}{\partial T}=\frac{d\left(\boldsymbol{r}_{\boldsymbol{i}}\right)}{d T} \boldsymbol{s}_{\boldsymbol{j}}+\boldsymbol{r}_{\boldsymbol{i}} \frac{d\left(\boldsymbol{s}_{\boldsymbol{j}}\right)}{d T}
$$

where.

$$
\begin{aligned}
& \frac{d\left(s_{j}\right)}{d T}=\frac{1}{l^{2}}\left[\frac{d y_{j+1}}{d T}-2 \frac{d y_{j}}{d T}+\frac{d y_{j-1}}{d T}\right], \frac{d\left(t_{i}\right)}{d T}=\frac{1}{2 l}\left[1+\left(\frac{y_{j+1}-y_{j-1}}{2 l}\right)^{2}\right]^{-1 / 2}\left(\frac{d y_{j+1}}{d T}-\frac{d y_{j-1}}{d T}\right) \\
& \frac{d\left(r_{i}\right)}{d T}=\frac{d\left(s_{i}\right)}{d T} E A\left(\sum_{1}^{k} t_{i}^{3}\right)^{-1}-\left(\sum_{1}^{k} t_{i}^{3}\right) \quad\left(\sum_{1}^{k} 3 t_{i}^{2} \frac{d\left(t_{i}\right)}{d T}\right) E A s_{i}, \frac{d \boldsymbol{Y}}{d T}=-\boldsymbol{K}_{1}^{-1} \frac{d \boldsymbol{K}_{1}}{d T} \boldsymbol{Y}
\end{aligned}
$$

where $i, j=1,2, \ldots, k$, and $k$ is the dimension of the stiffness matrix. The nonlinear stiffness matrix $K_{2}$ is related to the static deflection of the cable.

With Eq.(20), the cable force can be solved iteratively after obtaining the derivative of cable frequency. Suppose

$$
\gamma=\frac{d \zeta}{d T}=\frac{\Delta \zeta}{\Delta T}
$$

The initial cable force is assumed to be $T_{1}=T^{e v a}$, and the frequency $\zeta_{1, c}$ and frequency derivative $\gamma_{1}$ can be obtained according to Eq.(18) and Eq.(20), respectively.

The frequency error $\Delta \zeta_{1}$ is

$$
\Delta \zeta_{1}=\zeta_{m}-\zeta_{1, c}
$$

where $\zeta_{m}$ denotes the measured frequency.

The cable force error $\Delta T_{1}$ is

$$
\Delta T_{1}=\frac{\Delta \zeta_{1}}{\gamma_{1}}
$$

Then the cable force $T_{2}$ can be obtained as

$$
T_{2}=T_{1}+\Delta T_{1}
$$

The above iterative process is repeated until the convergent cable force value $\left(T_{i+1}\right)$ is obtained. It should be noted that this study mainly focuses on the effects of inclination angle on the in-plane frequency and the corresponding estimated cable force. Actually the inclination angle may also affect out-of-plane frequency and this would be further investigated.

\subsection{Numerical study}

The characteristic parameters $\lambda^{2}$ and $\xi$ are employed to reflect the sag and bending stiffness of the cable, respectively (Irvine and Caughey 1974). Specific as

$$
\begin{aligned}
\lambda^{2} & =\left(\frac{m g L}{T}\right)^{2} \frac{E A L}{T L_{e}} \\
\xi & =\sqrt{\frac{T}{E I}} L
\end{aligned}
$$

where $L_{e}$ denotes the effective length of the cable.

The ranges of the two parameters for more than $95 \%$ of cable-stayed bridges in the world are $\lambda^{2}<3.1$ and $\xi>50$ (Tabatabai et al. 1997). In other words, most cables belong to cables with small sag. This section aims to investigate the inclination angle effect on 
the modal parameters and estimated cable force for fixed-fixed and hinged-hinged cables with small sag and different bending stiffness.

Three cables as listed in Table 1 are employed in this example. The parameters of Cable I and Cable II are taken from Ref. (Kim et al. 2007; Ma 2017). Cable I, Cable II, and Cable III are deemed as cables with small bending stiffness, medium bending stiffness, and large bending stiffness, respectively. The cable is divided into 100 segments equally for analysis.

Natural frequency is an important parameter for cable force estimation in vibration based method. This section studies the effect of different inclination angle on natural frequency of hinged-hinged cable. The relation curves between the frequency and the inclination angle of the three cables are obtained by using the cable vibration theory expressed in Section 2 and shown in Fig. 3. The marked frequency values in the figure are the frequency values of the cable when the inclination angle are equal to $0^{\circ}, 30^{\circ}, 60^{\circ}$ and $90^{\circ}$, respectively. It can be seen that the fundamental frequency of cables with small sag (large, medium and small bending stiffness) decreases with inclination angle, and the maximum relative reduction is $8.53 \%$. When the frequency sensitivity based method is used to estimate the cable force (Kim et al. 2007; Ma 2017), mode shape of the cable is required during the iterative process. The first order maximum normalized mode shapes of three cables with different bending stiffness and inclination angles are calculated via the above mentioned method. The results indicated that the effects of inclination angle on mode shapes of the three cables are not obvious, thus they are not exhibition here for the space cause.

As the inclination angle affects the natural frequency of the cable, the cable force estimation accuracy would also be affected accordingly. The cable force estimation results of hinged-hinged cables $\left(30^{\circ}\right.$ and $\left.60^{\circ}\right)$ with and without considering the inclination angle effects are compared in Table 2. For the hinged-hinged cable, the maximum cable force estimation error is about $10 \%$ when the inclination angle effects are not considered. The results show that the influence of inclination on frequency, mode shape and cable force estimation accuracy for fixed-fixed cables is almost the same as the hingedhinged cable. The numerical examples clearly indicate the necessity of considering the inclination angle effect on the cable force estimation for inclined cables with small sag.

\section{Practical cable force estimation formulas for cables with known boundary}

The iterative method is not convenient for practical application as the process is too complicated. Therefore it is necessary to establish a practical formula for cable force estimation based on the fundamental frequency.

\subsection{Analysis of the cable frequency and characteristic parameters}

The relationship between the cable force, bending stiffness and frequency square for hinged-hinged cables with different inclination angle $\left(0^{\circ}, 30^{\circ}, 60^{\circ}\right.$ and $\left.90^{\circ}\right)$ are

Table 1 Physical and geometric parameters of the three cables

\begin{tabular}{lllllllll}
\hline Cable. & $\boldsymbol{\lambda}^{\mathbf{2}}$ & $\boldsymbol{\xi}$ & $\boldsymbol{m}(\mathbf{k g} / \boldsymbol{m})$ & $\boldsymbol{L}(\boldsymbol{m})$ & $\mathbf{H}_{\boldsymbol{M}}(\boldsymbol{M N})$ & $\boldsymbol{E}(\mathbf{G P a})$ & $\boldsymbol{A}\left(\times \mathbf{1 0}^{-\mathbf{3}} \mathbf{m}^{\mathbf{2}}\right)$ & $\boldsymbol{I}\left(\times \mathbf{1 0}^{-\mathbf{6}} \mathbf{m}^{\mathbf{4}}\right)$ \\
\hline Cable I & 0.79 & 605.5 & 400.0 & 100 & 2.9036 & 15.988 & 7.8507 & 4.9535 \\
Cable II & 1.41 & 50.5 & 400.0 & 100 & 26.1325 & 20,826 & 7.8633 & 4.9204 \\
Cable III & 2.76 & 10.7 & 400.0 & 100 & 90.0000 & $1,716,800$ & 7.6110 & 4.6097 \\
\hline
\end{tabular}




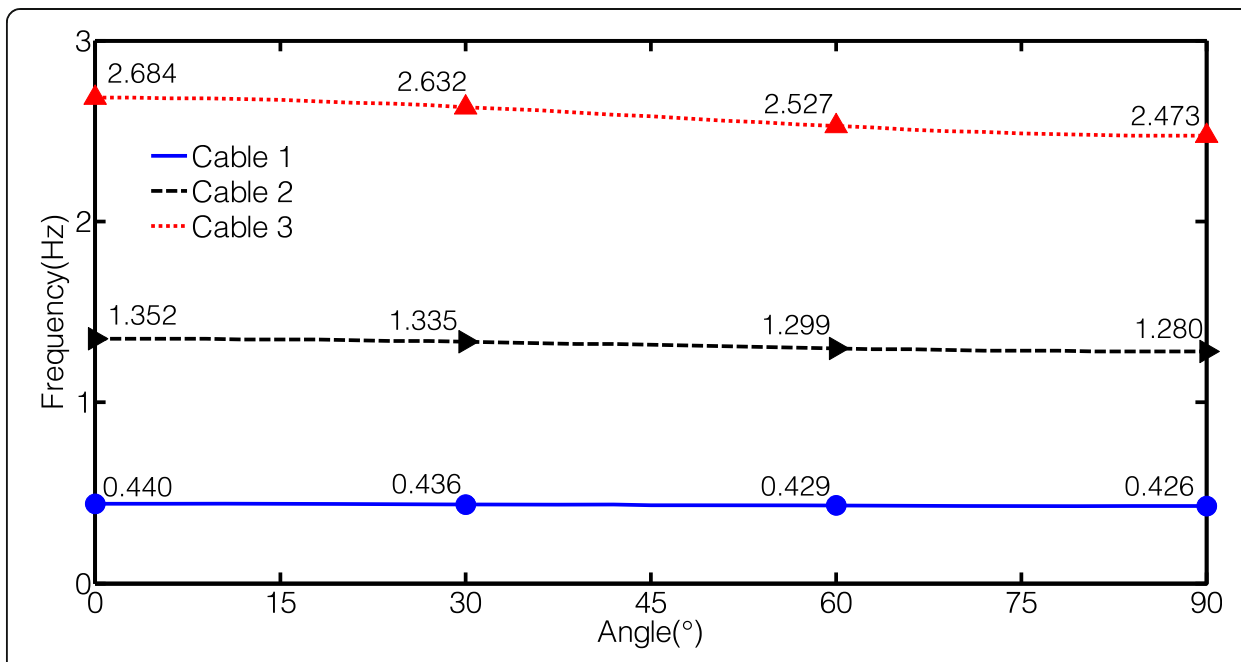

Fig. 3 Frequency-angel curve of hinged-hinged cables. (a) $0^{\circ}$. (b) $30^{\circ}$. (c) $60^{\circ}$. (d) $90^{\circ}$

calculated according to the above-mentioned method and shown in Fig. 4. It can be seen that bulge appears in the frequency of the cable with large sag when the cable force is small. With the increase of inclination angle, the bulge disappears gradually. When the inclination angle is $90^{\circ}$, no bulge can be found and obvious linear relationship can be found between the frequency square, cable force and bending stiffness. This is because the cable is not affected by the sag when the inclination angle is $90^{\circ}$, and the results conforms to the cable force estimation formula derived from the theory of hinged-hinged beam. With the increase of cable force, the frequency corresponding to small sag cable is nearly flat. However, due to the influence of inclination angle, the slope of the surface is varies with inclination angle. When the cable force continues to increase, the sag effect of the cable become very small, and the influence of inclination angle can be ignored. Thus the surface at each inclination angle is close to the theoretical formula derived from the theory of hinged-hinged beam.

For cables with large sag, the cable frequency first increases and then converges to a stable value with the increase of bending stiffness. It should be noted that the increase of inclination angle accelerates the convergence speed. For cables with small sag, the frequency surface of the cable increases gently. When the sag is extremely small, the bending stiffness has little influence on the frequency.

Table 2 Estimation results of a hinged-hinged cable with inclination of $30^{\circ}$ and $60^{\circ}$

\begin{tabular}{|c|c|c|c|c|c|c|c|}
\hline \multirow[t]{2}{*}{ Cable } & \multirow{2}{*}{$\begin{array}{l}\text { Angel } \\
\left({ }^{\circ}\right)\end{array}$} & \multirow{2}{*}{$\begin{array}{l}\mathrm{FF} \\
(\mathrm{Hz})\end{array}$} & \multirow{2}{*}{$\begin{array}{l}\text { RCF } \\
(\mathrm{MN})\end{array}$} & \multicolumn{2}{|l|}{ WCAE } & \multicolumn{2}{|l|}{ CAE } \\
\hline & & & & $\mathrm{EF}(\mathrm{MN})$ & RE (\%) & $\mathrm{EF}(\mathrm{MN})$ & RE (\%) \\
\hline \multirow[t]{2}{*}{ Cable I } & 30 & 0.436 & 2.9036 & 2.8447 & -2.028 & 2.9002 & -0.118 \\
\hline & 60 & 0.429 & 2.9036 & 2.7312 & -5.938 & 2.8999 & -0.128 \\
\hline \multirow[t]{2}{*}{ Cable II } & 30 & 1.335 & 26.13254 & 25.1504 & -3.758 & 26.1484 & 0.061 \\
\hline & 60 & 1.299 & 26.13254 & 22.9983 & -11.994 & 26.1427 & 0.039 \\
\hline \multirow[t]{2}{*}{ Cable III } & 30 & 2.632 & 90.0000 & 82.6892 & -8.123 & 89.9544 & -0.051 \\
\hline & 60 & 2.527 & 90.0000 & 79.1614 & -12.043 & 90.0070 & 0.008 \\
\hline
\end{tabular}

Note: $F F$ denotes fundamental frequency; $R C F$ denotes reference cable force; $R E$ denotes relative error; $E F$ denotes estimated force; WCAE denotes result without considering angel effect; and CAE denotes result considering angel effect 

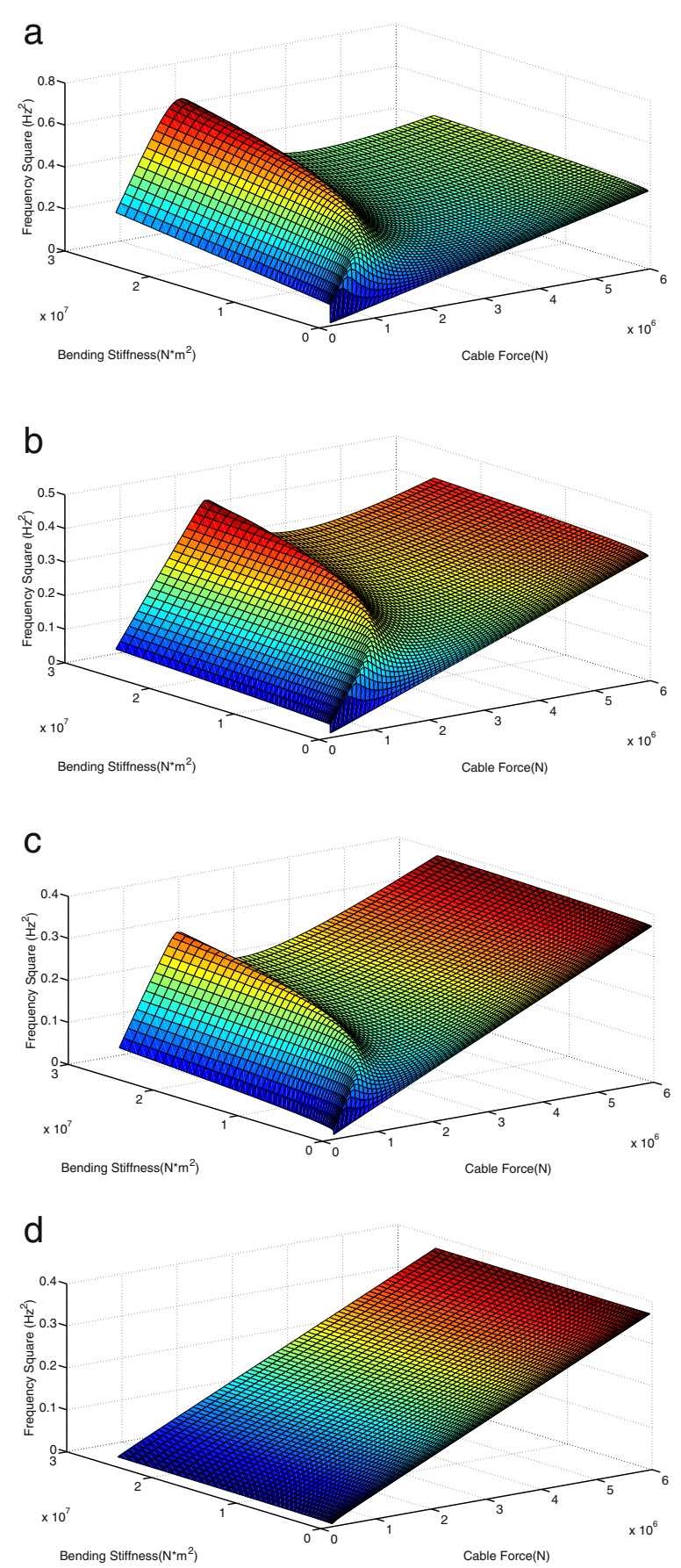

Fig. 4 Frequency square-cable parameters surface

\subsection{Practical formula for cable force estimation}

As pointed out in Section 3.1, most cables of cable-stayed bridges belong to cables with small sag $\left(\lambda^{2}<3.1\right)$. According to Section 4.1, the frequency square of cables with small sage is linearly related to the cable force and bending stiffness. However, the surface slope varies with inclination angle. When $\lambda^{2}<0.17$, cable force can be calculated according to the string theory without leading to obvious error (Ceballos and Prato 2008). Thus this section aims to establish the practical formula considering the 
inclination angle effect for fixed-fixed and hinged-hinged cables with small sag $\left(0.17<\lambda^{2}<3.1\right)$ based on curve fitting method.

Cable II with the parameters used in Section 3.2 is reused in this section. The dimensionless parameter $\lambda^{2}$ is restricted into the interval $[0.17,3.10]$ by adjusting the cable force value in Eq.(26). The interval of the dimensionless parameter $\xi$ is calculated via Eq.(27) by setting different bending stiffness of the cable (Fang and Wang 2012). Details about the parameters are listed in Table 3.

For the cables with large bending stiffness $(0<\xi<18)$, the relationship curve between cable force and frequency square with different inclination angles are obtained by using the cable vibration theory. Figure 5 shows the curves with inclination angles of $0^{\circ}, 30^{\circ}$, $60^{\circ}$ and $90^{\circ}$. It is observed that the relationship between the cable force and frequency square is linear and the curve slopes are different depend on the inclination angels. Refer to the Ref. (Huang et al. 2015), a linear function between the cable force and frequency square can be obtained by using the regression analysis.

$$
T=\alpha \cdot m L^{2} f_{1}^{2}-\beta \cdot \frac{E I}{L^{2}}
$$

where $\alpha$ and $\beta$ are fitting coefficients to be determined. Their values when the inclination angles are $0^{\circ}, 15^{\circ}, 30^{\circ}, 45^{\circ}, 60^{\circ}, 75^{\circ}$ and $90^{\circ}$ are calculated and compared in Table 4. It is observed that that the coefficients $\alpha$ and $\beta$ decrease with the inclination angle.

Suppose the function forms are $\alpha=a_{1} \cos \left(b_{1} \theta\right)+c_{1}$ and $\beta=a_{2} \cos \left(b_{2} \theta\right)+c_{2}$, the fitting curves of the two coefficients are plotted in Fig. 6. The coefficients $\alpha$ and $\beta$ obtained by linear regression analysis are:

$$
\begin{aligned}
& \alpha=0.201 \cos (2.038 \theta)+4.202 \\
& \beta=13.020 \cos (2.042 \theta)+22.960
\end{aligned}
$$

Thus the practical cable force estimation formula for hinged-hinged cables with large bending stiffness $(0<\xi<18)$ and small sag $\left(0.17<\lambda^{2}<3.10\right)$ can be acquired via substituting Eq.(29a) and Eq.(29b) into Eq.(28).

Following the same procedure, the practical cable force estimation formulas considering the inclination angle effect for hinged-hinged cables and fixed-fixed cables with small sag $\left(0.17<\lambda^{2}<3.10\right)$ can be obtained (Table 4 and Figs.7, 8, 9 and 10). The results can be summarized as

$$
T=\left[a_{1} \cos \left(b_{1} \theta\right)+\mathrm{c}_{1}\right] m L^{2} f_{1}^{2}-\left[a_{2} \cos \left(b_{2} \theta\right)+c_{2}\right] \frac{E I}{L^{2}}
$$

Table 3 Dimensionless parameter range

\begin{tabular}{lllll}
\hline $\begin{array}{l}\text { Cable Force } \\
(\boldsymbol{M N})\end{array}$ & $\begin{array}{l}\text { Bending Stiffness } \\
\left(\mathbf{M N} \cdot \mathbf{m}^{\mathbf{2}}\right)\end{array}$ & $\boldsymbol{\lambda}^{\mathbf{2}}$ & $\boldsymbol{\xi}$ & $\begin{array}{l}\text { Reference } \boldsymbol{\xi} \\
\text { (Ren et al. 2005) }\end{array}$ \\
\hline$[93.5,245]$ & 10,244 & {$[0.17,3.10]$} & {$[9.55,15.42]$} & {$[0,18]$} \\
{$[20,52]$} & 102.44 & {$[0.17,3.10]$} & {$[44.19,71.25]$} & {$[18,210]$} \\
{$[2,5.2]$} & 0.10244 & {$[0.17,3.10]$} & {$[441.85,712.46]$} & {$[210,+\infty]$} \\
\hline
\end{tabular}




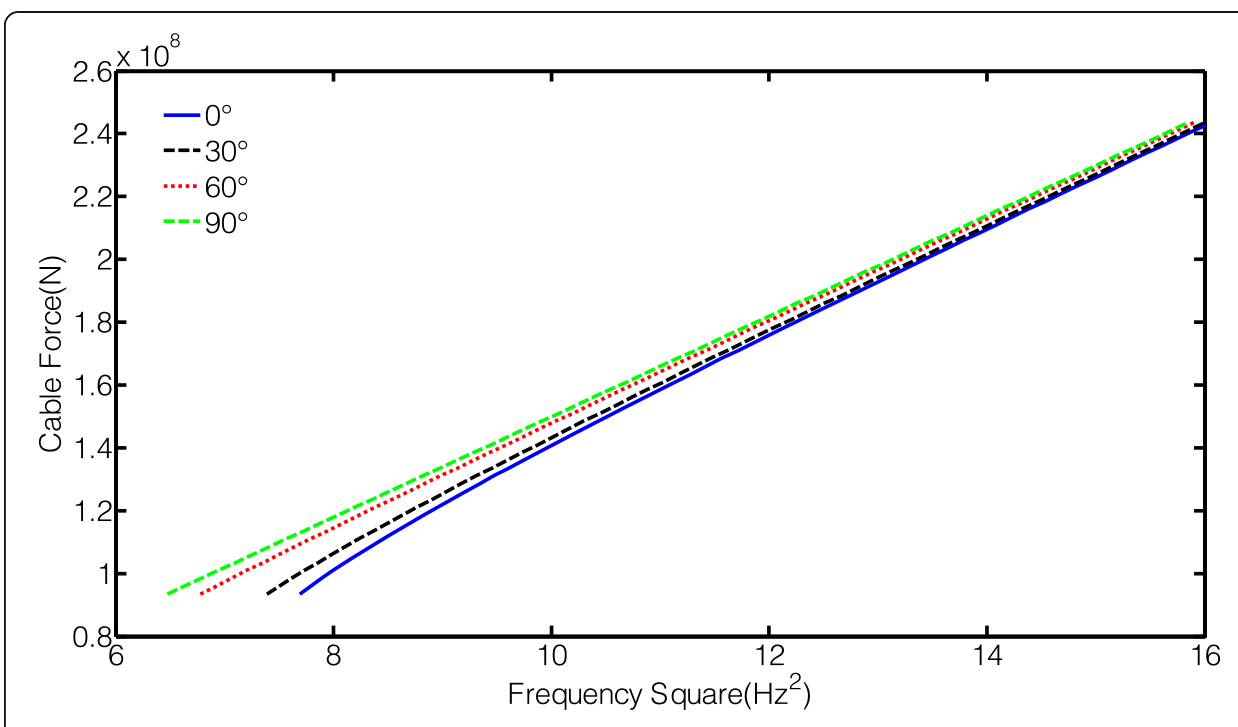

Fig. 5 Cable force-frequency square curve for hinged-hinged cable with large bending stiffness. (a) $a$. (b) $\beta$

And the coefficients $a_{1}, a_{2}, b_{1}, b_{2}, c_{1}$ and $c_{2}$ are listed in Table 5 .

\subsection{Numerical study}

This section aims to verify the effeteness and study the applicability of the practical formulas proposed in Section 4.2 by numerical examples. The relevant parameters of the three cables here are the same as in Section 3.2.

The estimated results of the hinged-hinged cable by the practical formula and the beam theory formula are compared in Table 6 . The results calculated by the practical formula considering the inclination angle effect achieve higher precision than that calculated by the beam theory formula. With the increase of the inclination angle, the differences between the results calculated by the two formulas decreases gradually. When the inclination angle is $90^{\circ}$, the errors of estimated cable force are both less than $1 \%$. This is because the influence of sag on cable force estimation decreases with the increase of inclination angle, and when the inclination angle is $90^{\circ}$ the cable becomes a suspension rod or suspension rope without sag. Table 7 compares the estimated results of the fixed-fixed cable by the practical formula and Ref. (Ma et al. 2021). The practical formula considering the inclination angle effect provides results with higher precision.

Table 4 Coefficients $a$ and $\beta$ of hinged-hinged cables

\begin{tabular}{|c|c|c|c|c|c|c|c|c|}
\hline Bending Stiffness & $\begin{array}{l}\text { Angle } \\
\left({ }^{\circ}\right)\end{array}$ & $0^{\circ}$ & $15^{\circ}$ & $30^{\circ}$ & $45^{\circ}$ & $60^{\circ}$ & $75^{\circ}$ & $90^{\circ}$ \\
\hline \multirow[t]{2}{*}{ Large bending stiffness } & $a$ & 4.4050 & 4.3750 & 4.2975 & 4.1950 & 4.0950 & 4.0250 & 4.000 \\
\hline & $\beta$ & 36.0885 & 34.1850 & 29.0992 & 22.4125 & 16.0089 & 11.4893 & 9.8689 \\
\hline \multirow[t]{2}{*}{ Medium Bending Stiffness } & $a$ & 4.5125 & 4.4750 & 4.3750 & 4.2450 & 4.1200 & 4.0325 & 4.0000 \\
\hline & $\beta$ & 682.0396 & 632.5486 & 500.7676 & 328.5738 & 165.0678 & 50.6429 & 9.8104 \\
\hline \multirow[t]{2}{*}{ Small Bending Stiffness } & $a$ & 4.5150 & 4.4775 & 4.3775 & 4.2450 & 4.1175 & 4.0275 & 3.9975 \\
\hline & $\beta$ & $6.7472 \mathrm{E} 4$ & $6.2503 \mathrm{E} 4$ & 4.9237E4 & 3.1842E4 & $1.5228 \mathrm{E} 4$ & $3.5503 \mathrm{E} 3$ & -627.668 \\
\hline
\end{tabular}




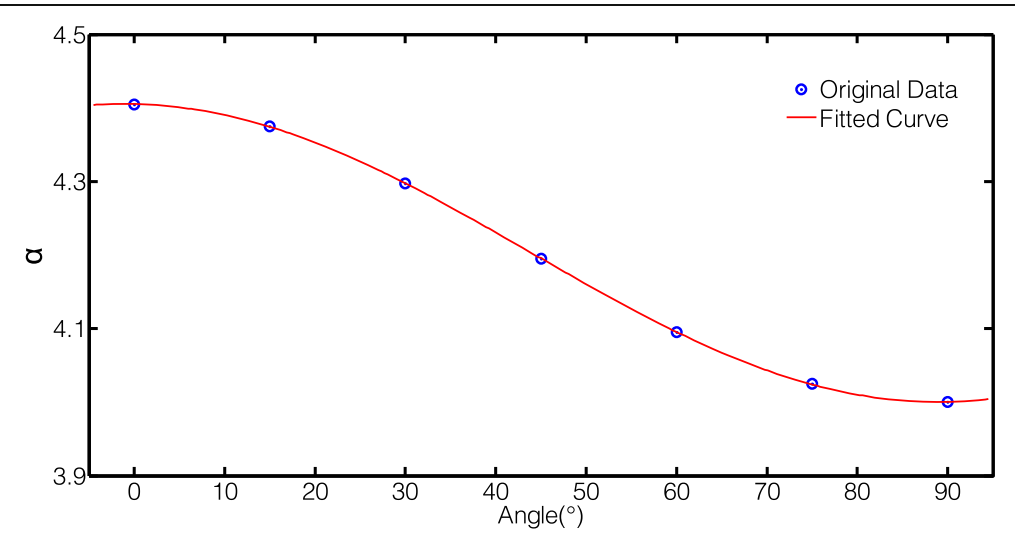

(a) $\alpha$

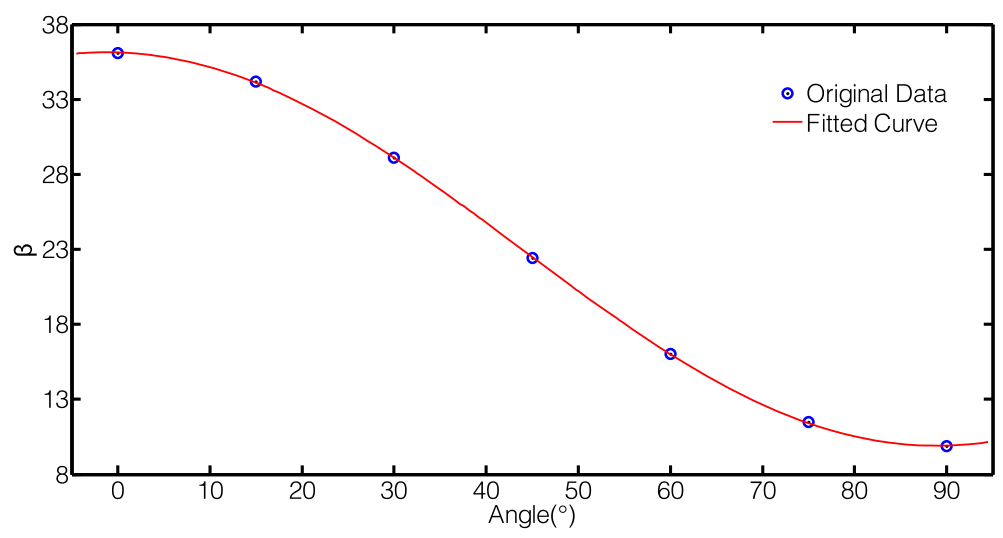

(b) $\beta$

Fig. 6 Coefficient-angle fitting curve for hinged-hinged cable with large bending stiffness

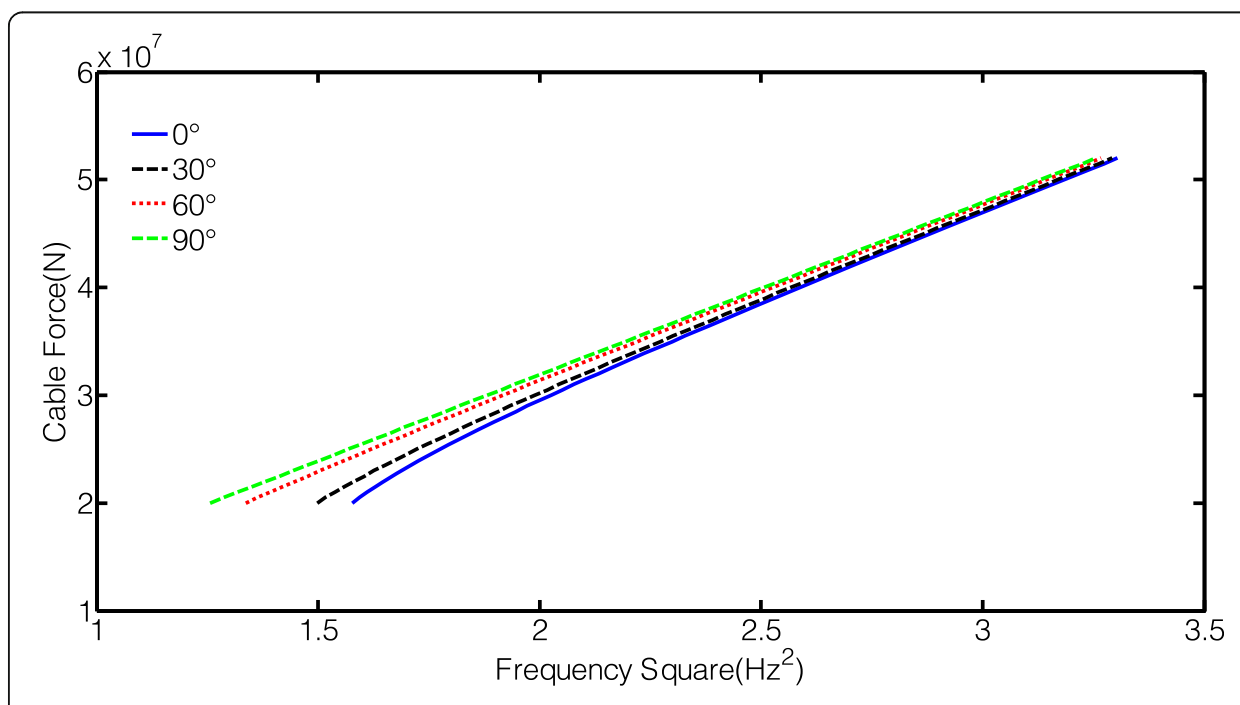

Fig. 7 Cable force-frequency square curve for hinged-hinged cable with medium bending stiffness. (a) $a$. (b) $\beta$ 


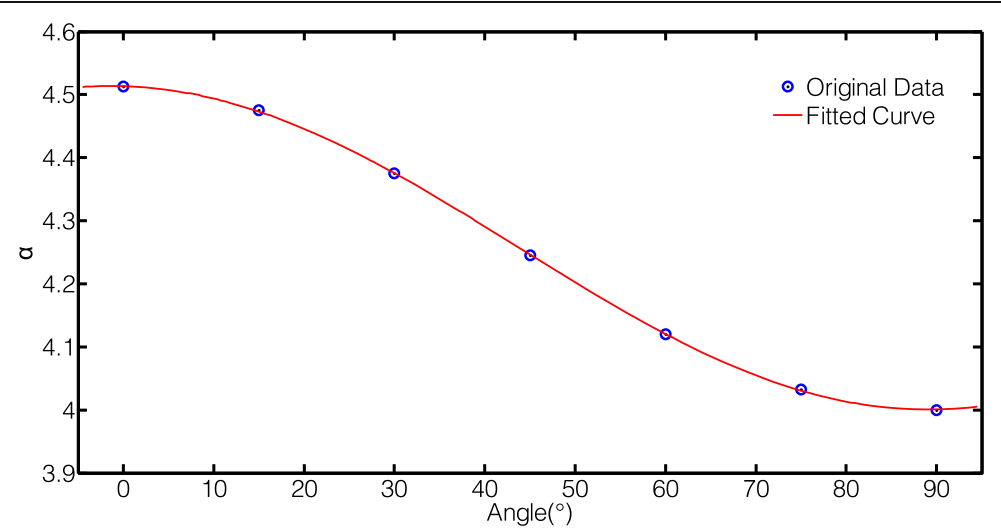

(a) $\alpha$

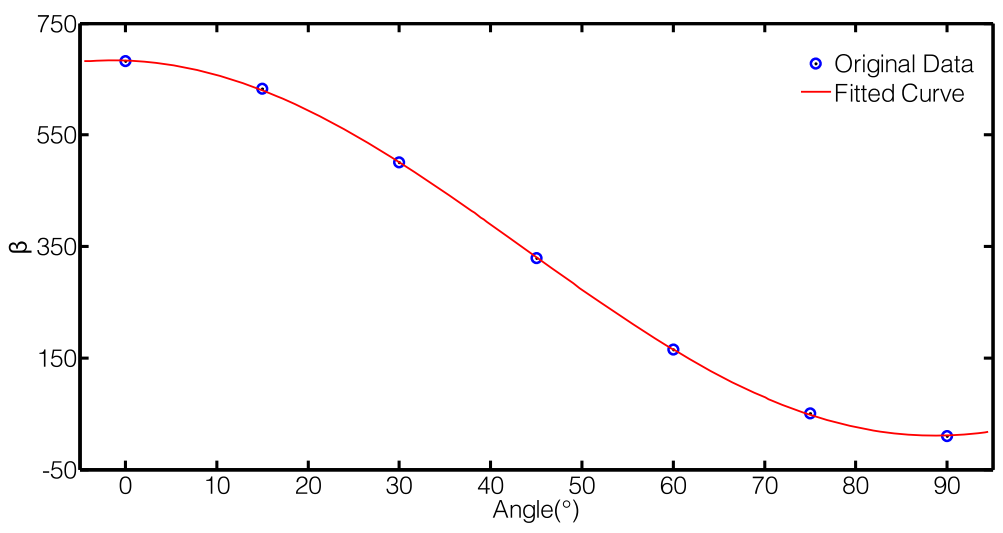

(b) $\beta$

Fig. 8 Coefficient-angle fitting curve for hinged-hinged cable with medium bending stiffness

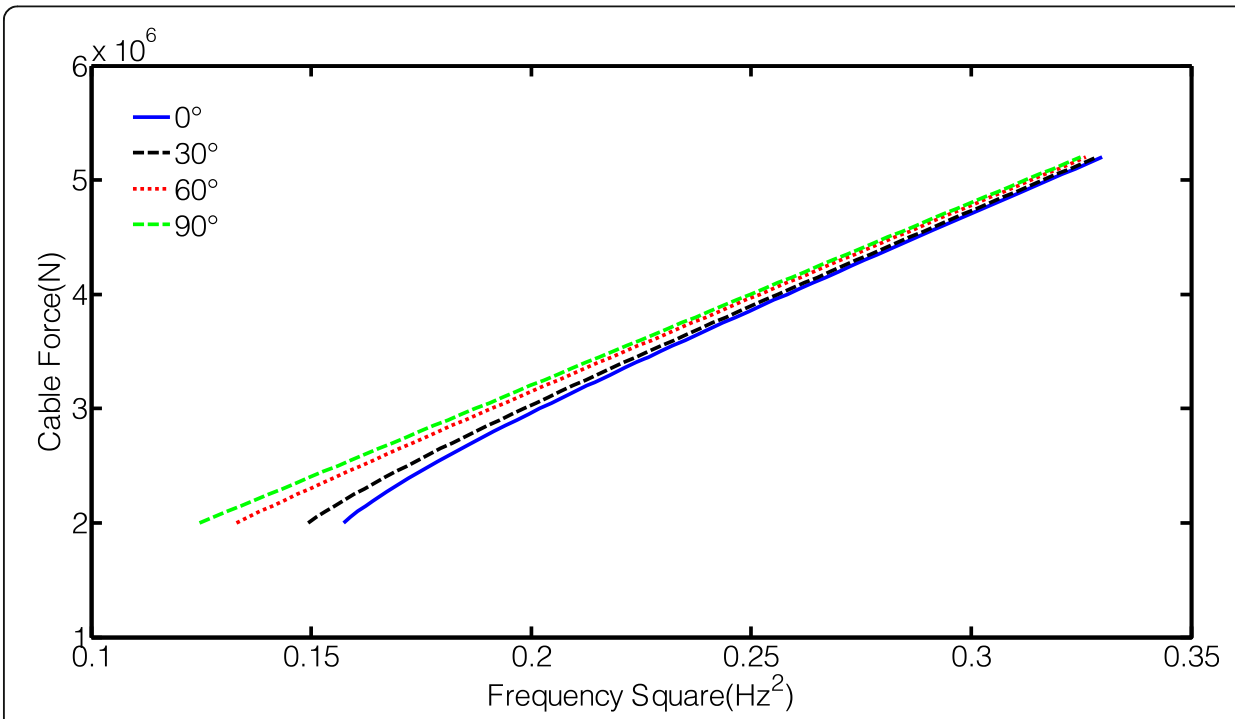

Fig. 9 Cable force-frequency square curve for hinged-hinged cable with small bending stiffness. (a) $a$. (b) $\beta$ 


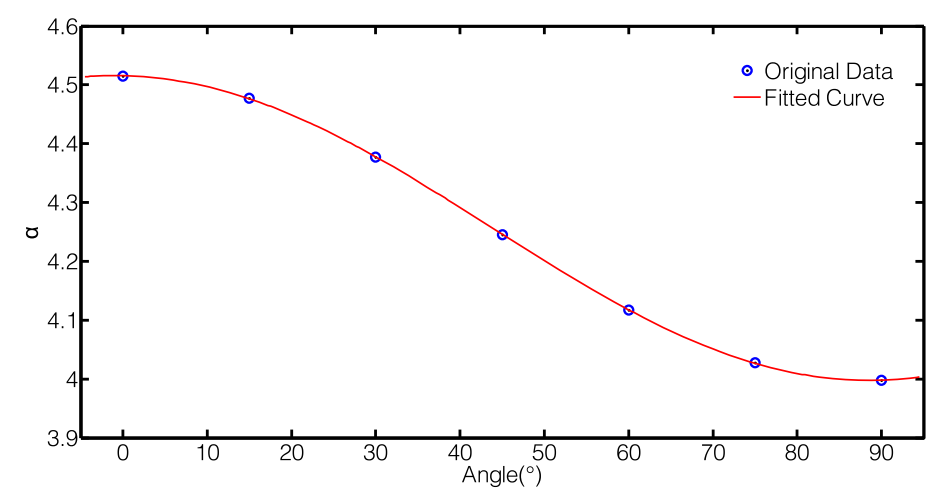

(a) $\alpha$

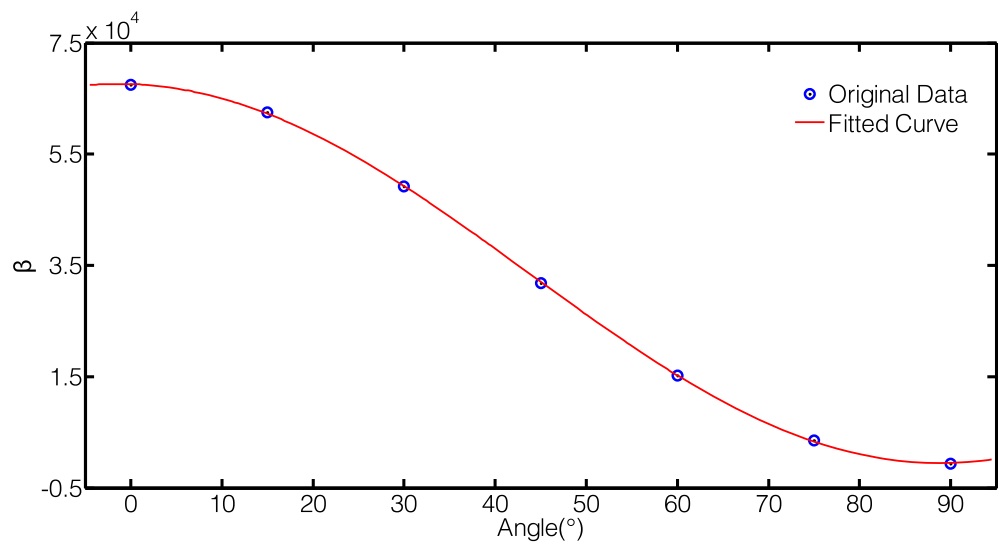

(b) $\beta$

Fig. 10 Coefficient-inclination fitting curve for hinged-hinged formula with small bending stiffness

Similarly to the hinged-hinged cable, the differences between the results calculated by the two formulas decrease gradually with the increase of the inclination angle.

Numerical examples reveal that the practical formula for cables with known boundary proposed in Section 4.2 can consider the inclination angle effects well and achieve higher accuracy for inclined cables with small sag. It should be noted that the accuracy of cable force estimation of Cable II is higher than that of Cable I and Cable III, which is caused by the fact the practical formula is fitted by the parameters of Cable II, which

Table 5 Coefficients of the practical formula for cable force estimation

\begin{tabular}{llllllll}
\hline Boundary & Bending stiffness & $\boldsymbol{a}_{\mathbf{1}}$ & $\boldsymbol{b}_{\mathbf{1}}$ & $\boldsymbol{c}_{\mathbf{1}}$ & $\boldsymbol{a}_{\mathbf{2}}$ & $\boldsymbol{b}_{\mathbf{2}}$ & $\boldsymbol{c}_{\mathbf{2}}$ \\
\hline Hinged-Hinged Cable & Large bending stiffness $(0 \leq \xi \leq 18)$ & 0.201 & 2.038 & 4.202 & 13.020 & 2.042 & 22.960 \\
& Medium bending stiffness & 0.254 & 2.042 & 4.256 & 336.000 & 2.000 & 338.500 \\
& $(18<\xi \leq 210)$ & & & & & & \\
& Small bending stiffness $(\xi>210)$ & 0.258 & 2.045 & 4.256 & $34,040.000$ & 2.000 & $32,740.000$ \\
Fixed-Fixed Cable & Large bending stiffness $(0 \leq \xi \leq 18)$ & 0.067 & 2.031 & 3.590 & 6.028 & 2.000 & 56.100 \\
& Medium bending stiffness & 0.203 & 2.044 & 4.089 & 285.200 & 2.000 & 418.000 \\
& $(18<\xi \leq 210)$ & & & & & & \\
& Small bending stiffness $(\xi>210)$ & 0.259 & 2.038 & 4.255 & $34,080.000$ & 2.000 & 3340.000 \\
\hline
\end{tabular}


Table 6 Force estimation results of hinged-hinged cables

\begin{tabular}{|c|c|c|c|c|c|c|c|}
\hline \multirow[t]{2}{*}{ Cable } & \multirow{2}{*}{$\begin{array}{l}\text { Angle } \\
\left({ }^{\circ}\right)\end{array}$} & \multirow{2}{*}{$\begin{array}{l}\mathrm{FF} \\
(H Z)\end{array}$} & \multirow{2}{*}{$\begin{array}{l}\text { RC F } \\
(M N)\end{array}$} & \multicolumn{2}{|c|}{ Proposed Method } & \multicolumn{2}{|c|}{ Beam Theory } \\
\hline & & & & $\mathrm{EF}(M N)$ & RE (\%) & $\mathrm{EF}(M N)$ & RE (\%) \\
\hline \multirow[t]{4}{*}{ Cable I } & 0 & 0.440 & \multirow[t]{4}{*}{2.9036} & 2.9665 & 2.16 & 3.0975 & 6.68 \\
\hline & 30 & 0.436 & & 2.9360 & 1.12 & 3.0415 & 4.75 \\
\hline & 60 & 0.429 & & 2.9062 & 0.09 & 2.9446 & 1.41 \\
\hline & 90 & 0.426 & & 2.9132 & 0.33 & 2.9035 & -0.002 \\
\hline \multirow[t]{4}{*}{ Cable II } & 0 & 1.352 & \multirow[t]{4}{*}{26.1325} & 26.0671 & -0.25 & 29.1454 & 11.53 \\
\hline & 30 & 1.335 & & 26.0232 & -0.42 & 28.4145 & 8.73 \\
\hline & 60 & 1.299 & & 26.0573 & -0.29 & 26.8973 & 2.93 \\
\hline & 90 & 1.280 & & 26.2042 & 0.27 & 26.1133 & -0.07 \\
\hline \multirow[t]{4}{*}{ Cable III } & 0 & 2.684 & \multirow[t]{4}{*}{90.0000} & 98.3759 & 9.31 & 107.4428 & 19.38 \\
\hline & 30 & 2.632 & & 95.9780 & 6.64 & 103.0199 & 14.47 \\
\hline & 60 & 2.527 & & 91.9424 & 2.16 & 94.3527 & 4.84 \\
\hline & 90 & 2.473 & & 89.9829 & -0.02 & 90.0327 & 0.04 \\
\hline
\end{tabular}

Note: $F F$ denotes fundamental frequency; $R C F$ denotes reference cable force; $R E$ denotes relative error; $E F$ denotes estimated force

manifests that the practical formula can be established according to the concerned cable when higher precision is required in practical application.

\section{Practical tension estimation formula for cables with unknown boundary}

\subsection{Boundary effects on the cable frequency}

The actual boundary condition of a cable is between the hinged and fixed, and the anchorage end of the cable always bears part of the bending moment. Therefore, the boundary conditions at both ends of the cable are assumed to be elastic embedded boundary, and the rotational stiffness at both ends is assumed to be $K_{\text {rot }-1}$ and $K_{\text {rot-2 }}$, as shown

Table 7 Force estimation results of fixed-fixed cables

\begin{tabular}{|c|c|c|c|c|c|c|c|}
\hline \multirow[t]{2}{*}{ Cable } & \multirow{2}{*}{$\begin{array}{l}\text { Angle } \\
\left({ }^{\circ}\right)\end{array}$} & \multirow{2}{*}{$\begin{array}{l}\mathrm{FF} \\
(H Z)\end{array}$} & \multirow{2}{*}{$\begin{array}{l}\text { RC F } \\
(M N)\end{array}$} & \multicolumn{2}{|c|}{ Proposed Method } & \multicolumn{2}{|c|}{ Ref. (Zui et al.1996) } \\
\hline & & & & $\mathrm{EF}(M N)$ & RE (\%) & $\mathrm{EF}(M N)$ & RE (\%) \\
\hline \multirow[t]{4}{*}{ Cable I } & 0 & 0.440 & 2.9036 & 2.9605 & 1.96 & 3.0758 & 5.93 \\
\hline & 30 & 0.437 & & 2.9458 & 1.45 & 3.0338 & 4.49 \\
\hline & 60 & 0.430 & & 2.9150 & 0.39 & 2.9371 & 1.15 \\
\hline & 90 & 0.426 & & 2.9065 & 0.10 & 2.8825 & -0.73 \\
\hline \multirow[t]{4}{*}{ Cable II } & 0 & 1.392 & 26.1325 & 26.0628 & -0.27 & 28.5004 & 9.06 \\
\hline & 30 & 1.377 & & 26.0094 & -0.47 & 27.8626 & 6.62 \\
\hline & 60 & 1.347 & & 26.0602 & -0.28 & 26.6085 & 1.82 \\
\hline & 90 & 1.332 & & 26.2208 & 0.34 & 25.9923 & -0.54 \\
\hline \multirow[t]{4}{*}{ Cable III } & 0 & 3.123 & 90.0000 & 93.4616 & 3.85 & 98.2242 & 9.14 \\
\hline & 30 & 3.101 & & 92.5151 & 2.79 & 96.3291 & 7.03 \\
\hline & 60 & 3.056 & & 90.7268 & 0.81 & 92.4945 & 2.77 \\
\hline & 90 & 3.034 & & 90.0424 & 0.05 & 90.6402 & 0.71 \\
\hline
\end{tabular}

Note: $F F$ denotes fundamental frequency; $R C F$ denotes reference cable force; $R E$ denotes relative error; $E F$ denotes estimated force 
in Fig. 2. According to the classical mechanics theory, for the fixed-fixed and fixed-hinged beam, unit rotation at the fixed end leads to 0 and $2 i(i=E I / l)$ bending moment at the hinged end and fixed end, respectively. Therefore, to produce a unit angle at the elastic embedded boundary, the required bending moment is between 0 and $2 i$.

The three cables mentioned in Section 3.2 are retaken as examples. The frequencies of three boundary conditions (hinged-hinged, fixed-fixed, and elastic embedded boundary) with different inclination angles are calculated according to the cable vibration theory and shown in Table 8 . The rotational stiffness of the two ends of the elastic embedded boundary are equal, i.e., $K_{\text {rot-1 }}=K_{\text {rot-2 }}$. The different elastic embedded boundaries are set to be $K_{r o t}^{\prime}=0.5 E I / l, K_{r o t}^{\prime \prime}=E I / l$, and $K_{r o t}^{\prime \prime \prime}=1.5 E I / l$, respectively.

\subsection{Cable force estimation process}

Taken Cable II as an example, this section proposes a cable force estimation process considering the influence of the inclination angle and unknown boundary conditions. The coefficient reflecting the elastic embedded boundary is firstly calculated by interpolation method based on practical formulas for fixed-fixed and hinged-hinged cables. Then the cable force taking into account the influence of the inclination angle and unknown boundary conditions is obtained through iteration.

According to Eq.(28), the practical force estimation formulas for cables under different boundary conditions can be expressed in the same form.

$$
T=\left[a_{1} \cos \left(b_{1} \theta\right)+c_{1}\right] m L^{2} f_{1}^{2}-\left[a_{2} \cos (2.000 \theta)+c_{2}\right] \frac{E I}{L^{2}}
$$

where $a_{1}, b_{1}, c_{1}, a_{2}$, and $c_{2}$ are coefficients to be determined according to the boundary conditions.

Table 8 Frequencies calculated using different boundary conditions

\begin{tabular}{llllll}
\hline Cable & Boundary Condition & $\mathbf{0}^{\circ}$ & $\mathbf{3 0}^{\circ}$ & $\mathbf{6 0}^{\circ}$ & $\mathbf{9 0}^{\circ}$ \\
\hline Cable I & Hinged & 0.440 & 0.436 & 0.429 & 0.426 \\
& Elastic $K_{\text {rot }}^{\prime}$ & 0.440 & 0.436 & 0.429 & 0.426 \\
& Elastic $K_{\text {rot }}^{\prime \prime}$ & 0.440 & 0.436 & 0.429 & 0.426 \\
& Elastic $K_{\text {rot }}^{\prime \prime \prime}$ & 0.440 & 0.436 & 0.429 & 0.426 \\
& Fixed & 0.440 & 0.437 & 0.430 & 0.426 \\
Cable II & Hinged & 1.352 & 1.335 & 1.299 & 1.280 \\
& Elastic $K_{\text {rot }}^{\prime}$ & 1.370 & 1.355 & 1.322 & 1.306 \\
& Elastic $K_{\text {rot }}^{\prime \prime}$ & 1.377 & 1.362 & 1.330 & 1.314 \\
& Elastic $K_{\text {rot }}^{\prime \prime \prime}$ & 1.381 & 1.366 & 1.334 & 1.317 \\
& Fixed & 1.392 & 1.377 & 1.347 & 1.332 \\
Cable III & Hinged & 2.684 & 2.632 & 2.527 & 2.472 \\
& Elastic $K_{\text {rot }}^{\prime}$ & 3.012 & 2.987 & 2.936 & 2.911 \\
& Elastic $K_{\text {rot }}^{\prime \prime \prime}$ & 3.060 & 3.036 & 2.989 & 2.965 \\
& Elastic $K_{\text {rot }}^{\prime \prime \prime}$ & 3.079 & 3.056 & 3.009 & 2.986 \\
& Fixed & 3.123 & 3.101 & 3.056 & 3.033 \\
\hline
\end{tabular}


Given that the elastic embedded boundary is between hinged and fixed, the average of the coefficients in practical formulas for hinged-hinged and fixed-fixed ends (Table 5) is employed as the coefficients to estimate the cable force $T_{0}$, specific as

$$
\begin{aligned}
T_{0} & =\left[\frac{0.25+0.203}{2} \cos \left(\frac{2.042+2.044}{2} \theta\right)+\frac{4.256+4.089}{2}\right] m L^{2} f_{1}^{2} \\
& -\left[\frac{336.000+285.200}{2} \cos (2.000 \theta)+\frac{338.500+418.000}{2}\right] \frac{E I}{L^{2}} \\
= & {[0.2285 \cos (2.0430 \theta)+4.1725]-[310.600 \cos (2.000 \theta)+378.250] }
\end{aligned}
$$

Then the estimated cable force $T_{0}$ is substituted into Eq.(29a) and Eq.(29b) to obtain the frequencies $\left(f_{1 j}\right.$ and $\left.f_{1 g}\right)$ of the cable with hinged-hinged and fixed-fixed ends, as

$$
\begin{aligned}
& f_{1 j}=\frac{1}{L \sqrt{\alpha_{j} m}} \sqrt{T_{0}+\beta_{j} \frac{E I}{L^{2}}} \\
& f_{1 g}=\frac{1}{L \sqrt{\alpha_{g} m}} \sqrt{T_{0}+\beta_{g} \frac{E I}{L^{2}}}
\end{aligned}
$$

The coefficients reflecting the elastic embedded boundary are obtained according to the interpolation formula (Eq.(34))

$$
\begin{aligned}
& a_{1}=0.254-0.051 \frac{f_{1}-f_{1 j}}{f_{1 g}-f_{1 j}} \\
& b_{1}=2.042+0.002 \frac{f_{1}-f_{1 j}}{f_{1 g}-f_{1 j}} \\
& c_{1}=4.256-0.167 \frac{f_{1}-f_{1 j}}{f_{1 g}-f_{1 j}} \\
& a_{2}=336.000-50.800 \frac{f_{1}-f_{1 j}}{f_{1 g}-f_{1 j}} \\
& c_{2}=338.500+79.500 \frac{f_{1}-f_{1 j}}{f_{1 g}-f_{1 j}}
\end{aligned}
$$

Finally the cable force $\left(T_{1}\right)$ is calculated via Eq.(31). If $\left(T_{1}-T_{0}\right) / T>1 \%$, the above process is repeated until $\left(T_{i}-T_{i-1}\right) / T_{i-1}<1 \%$, and the $T_{i}$ is the estimated cable force. Normally $T_{1}$ can satisfy this terminate circulation condition.

\subsection{Numerical study}

Section 5.1 discussed the influence of boundary conditions on the frequency of cables with different bending stiffness, and Section 5.2 presented a cable force estimation method that takes into account the influence of the inclination angle and unknown boundary conditions. This section will analyze the influence of boundary conditions on cable force estimation for cables with different bending stiffness, and verify the effectiveness of cable force estimation method under unknown boundary conditions. The relevant parameters of cables are the same as in Section 5.1, and the cable force estimation processes of Cable I and Cable III are the same as that of Cable II. The force estimation results are presented in Table 9, Table 10, and Table 11, respectively. 
Table 9 Force estimation result of Cable I

\begin{tabular}{|c|c|c|c|c|c|c|c|c|c|}
\hline \multirow[t]{2}{*}{ EC } & \multirow{2}{*}{$\begin{array}{l}\text { Angle } \\
\left({ }^{\circ}\right)\end{array}$} & \multirow{2}{*}{$\begin{array}{l}\mathrm{FF} \\
(\mathrm{Hz})\end{array}$} & \multirow{2}{*}{$\begin{array}{l}\text { RCF } \\
(M N)\end{array}$} & \multicolumn{2}{|c|}{ Hinged-Hinged } & \multicolumn{2}{|c|}{ Fixed-Fixed } & \multicolumn{2}{|c|}{ Identified } \\
\hline & & & & $T_{j}(M N)$ & $\mathrm{RE}(\%)$ & $T_{g}(M N)$ & $\operatorname{RE}(\%)$ & $T_{1}(M N)$ & $\mathrm{RE}(\%)$ \\
\hline \multirow[t]{4}{*}{$\overline{K_{\text {rot }}^{\prime}}$} & 0 & 0.440 & 2.9036 & 2.8705 & -1.14 & 2.8654 & -1.31 & 2.8680 & -1.23 \\
\hline & 30 & 0.436 & & 2.8655 & -1.31 & 2.8600 & -1.50 & 2.8627 & -1.41 \\
\hline & 60 & 0.429 & & 2.8848 & -0.65 & 2.8790 & -0.85 & 2.8819 & -0.75 \\
\hline & 90 & 0.426 & & 2.9137 & 0.35 & 2.9086 & 0.17 & 2.9112 & 0.26 \\
\hline \multirow{4}{*}{$K_{\text {rot }}^{\prime \prime}$} & 0 & 0.440 & 2.9036 & 2.8705 & -1.14 & 2.8654 & -1.31 & 2.8680 & -1.23 \\
\hline & 30 & 0.436 & & 2.8655 & -1.31 & 2.8600 & -1.50 & 2.8627 & -1.41 \\
\hline & 60 & 0.429 & & 2.8848 & -0.65 & 2.8790 & -0.85 & 2.8819 & -0.75 \\
\hline & 90 & 0.426 & & 2.9137 & 0.35 & 2.9086 & 0.17 & 2.9112 & 0.26 \\
\hline \multirow[t]{4}{*}{$K_{\text {rot }}^{\prime \prime \prime}$} & 0 & 0.440 & 2.9036 & 2.8705 & -1.14 & 2.8654 & -1.31 & 2.8680 & -1.23 \\
\hline & 30 & 0.436 & & 2.8655 & -1.31 & 2.8600 & -1.50 & 2.8627 & -1.41 \\
\hline & 60 & 0.429 & & 2.8848 & -0.65 & 2.8790 & -0.85 & 2.8819 & -0.75 \\
\hline & 90 & 0.426 & & 2.9137 & 0.35 & 2.9086 & 0.17 & 2.9112 & 0.26 \\
\hline
\end{tabular}

Note: $E B$ denotes elastic boundary; FF denotes fundamental frequency; $R C F$ denotes reference cable force; $R E$ denotes relative error

For the cables with small bending stiffness (Cable I), the calculated frequencies under different elastic embedded boundaries and the same inclination angle are the same, thus the corresponding cable forces estimated according to the frequency and inclination angle are identical (Table 9). The relative errors of the cables under three different boundary conditions are almost the same and less than 2\% (Table 9). In other words, the boundary conditions have little influence on the cables with small bending stiffness. In actual application, the boundary conditions of cables can be treated as hinged-hinged or fixed-fixed for simple.

As shown in Table 10 and Table 11, the boundary conditions have significant influence on the force estimation for the cables with medium bending stiffness (Cable II) and large bending stiffness (Cable III). The estimation results obtained by the method proposed in this section are more accurate than that obtained by the method under

Table 10 Force estimation result of Cable II

\begin{tabular}{|c|c|c|c|c|c|c|c|c|c|}
\hline \multirow[t]{2}{*}{ EC } & \multirow{2}{*}{$\begin{array}{l}\text { Angle } \\
\left({ }^{\circ}\right)\end{array}$} & \multirow{2}{*}{$\begin{array}{l}\text { FF } \\
(H z)\end{array}$} & \multirow{2}{*}{$\begin{array}{l}\text { RCF } \\
(M N)\end{array}$} & \multicolumn{2}{|c|}{ Hinged-Hinged } & \multicolumn{2}{|c|}{ Fixed-Fixed } & \multicolumn{2}{|c|}{ Estimated } \\
\hline & & & & $T_{j}(M N)$ & $\mathrm{RE}(\%)$ & $T_{g}(M N)$ & $\mathrm{RE}(\%)$ & $T_{1}(M N)$ & $\mathrm{RE}(\%)$ \\
\hline \multirow[t]{4}{*}{$K_{\text {rot }}^{\prime}$} & 0 & 1.370 & 26.1325 & 26.9495 & 3.13 & 25.0188 & -4.26 & 26.0010 & -0.50 \\
\hline & 30 & 1.355 & & 26.9647 & 3.18 & 25.0026 & -4.32 & 25.9978 & -0.52 \\
\hline & 60 & 1.322 & & 27.0513 & 3.52 & 24.9984 & -4.34 & 26.0330 & -0.38 \\
\hline & 90 & 1.306 & & 27.2820 & 4.40 & 25.1553 & -3.74 & 26.2238 & 0.35 \\
\hline \multirow[t]{4}{*}{$K_{\text {rot }}^{\prime \prime}$} & 0 & 1.377 & 26.1325 & 27.2964 & 4.45 & 25.3490 & -3.00 & 26.3397 & 0.79 \\
\hline & 30 & 1.362 & & 27.2978 & 4.46 & 25.3211 & -3.11 & 26.3237 & 0.73 \\
\hline & 60 & 1.330 & & 27.4009 & 4.85 & 25.3361 & -3.05 & 26.3768 & 0.93 \\
\hline & 90 & 1.314 & & 27.6176 & 5.68 & 25.4811 & -2.49 & 26.5546 & 1.62 \\
\hline \multirow[t]{4}{*}{$K_{\text {rot }}^{\prime \prime \prime}$} & 0 & 1.381 & 26.1325 & 27.4954 & 5.22 & 25.5384 & -2.27 & 26.5340 & 1.54 \\
\hline & 30 & 1.366 & & 27.4889 & 5.19 & 25.5038 & -2.41 & 26.5107 & 1.45 \\
\hline & 60 & 1.334 & & 27.5765 & 5.53 & 25.5058 & -2.40 & 26.5949 & 1.60 \\
\hline & 90 & 1.317 & & 27.7439 & 6.17 & 25.6038 & -2.02 & 26.4739 & 1.29 \\
\hline
\end{tabular}

Note: $E B$ denotes elastic boundary; $F F$ denotes fundamental frequency; $R C F$ denotes reference cable force; $R E$ denotes relative error 
Table 11 Force estimation result of Cable III

\begin{tabular}{|c|c|c|c|c|c|c|c|c|c|}
\hline \multirow[t]{2}{*}{ EC } & \multirow{2}{*}{$\begin{array}{l}\text { Angle } \\
\left({ }^{\circ}\right)\end{array}$} & \multirow{2}{*}{$\begin{array}{l}\mathrm{FF} \\
(\mathrm{Hz})\end{array}$} & \multirow{2}{*}{$\begin{array}{l}\text { RCF } \\
(M N)\end{array}$} & \multicolumn{2}{|c|}{ Hinged-Hinged } & \multicolumn{2}{|c|}{ Fixed-Fixed } & \multicolumn{2}{|c|}{ Estimated } \\
\hline & & & & $T_{j}(M N)$ & $\mathrm{RE}(\%)$ & $T_{g}(M N)$ & $\mathrm{RE}(\%)$ & $T_{1}(M N)$ & $\mathrm{RE}(\%)$ \\
\hline \multirow[t]{4}{*}{$\overline{K_{\text {rot }}^{\prime}}$} & 0 & 3.012 & 90.0000 & 127.1397 & 41.27 & 80.7797 & -10.24 & 91.0980 & 1.22 \\
\hline & 30 & 2.987 & & 127.0212 & 41.13 & 80.3083 & -10.77 & 91.1700 & 1.30 \\
\hline & 60 & 2.936 & & 127.2972 & 41.44 & 79.4885 & -11.68 & 90.9450 & 1.05 \\
\hline & 90 & 2.911 & & 128.0851 & 42.32 & 79.2526 & -11.94 & 91.0080 & 1.12 \\
\hline \multirow[t]{4}{*}{$K_{\text {rot }}^{\prime \prime}$} & 0 & 3.060 & 90.0000 & 132.2906 & 46.99 & 85.0960 & -5.45 & 91.3140 & 1.46 \\
\hline & 30 & 3.036 & & 132.1089 & 46.79 & 84.6334 & -5.96 & 91.2690 & 1.41 \\
\hline & 60 & 2.989 & & 132.4438 & 47.16 & 83.9941 & -6.67 & 91.8450 & 2.05 \\
\hline & 90 & 2.965 & & 133.1648 & 47.96 & 83.7579 & -6.94 & 94.0050 & 4.45 \\
\hline \multirow[t]{4}{*}{$K_{\text {rot }}^{\prime \prime \prime}$} & 0 & 3.079 & 90.0000 & 134.3520 & 49.28 & 86.8234 & -3.53 & 92.2320 & 2.48 \\
\hline & 30 & 3.056 & & 134.2093 & 49.12 & 86.4190 & -3.98 & 92.1510 & 2.39 \\
\hline & 60 & 3.009 & & 134.4098 & 49.34 & 85.7153 & -4.76 & 92.9430 & 3.27 \\
\hline & 90 & 2.986 & & 135.1655 & 50.18 & 85.5323 & -4.96 & 93.3660 & 3.74 \\
\hline
\end{tabular}

Note: $E B$ denotes elastic boundary; $F F$ denotes fundamental frequency; $R C F$ denotes reference cable force; $R E$ denotes relative error

hinged-hinged and fixed-fixed boundary conditions and the relative errors are less than $2 \%$ and $5 \%$ for the Cable II and Cable III, respectively. The boundary conditions of cables with medium bending stiffness and large bending stiffness cannot be simplified as hinged-hinged or fixed-fixed ends, and should be treated as unknown boundary conditions in actual application.

\section{Conclusion}

Horizontal cable model are normally employed in the most of the existing cable force estimation methods without considering the influence of inclination angle. This study investigated the influence of inclination angle on cable force estimation accuracy and provided practical formulas accordingly. The main conclusions are as follows:

(1) The fundamental frequency of a cable with small sag decreased with the increase of the inclination angle. Significant errors could be found when the fundamental frequency was applied to estimate the cable force without considering the inclination angle effects.

(2) The proposed practical force estimation formulas for cables with fixed-fixed and hinged-hinged cables could consider the inclination angle effects well and achieve higher accuracy for inclined cables with small sag. Besides, the practical formula could be established according to the concerned cable when higher precision was required in practical application.

(3) The proposed practical force estimation formulas for cables under unknown boundary conditions possessed high precision and superior practicability. The influence of boundary conditions on the accuracy of cable force estimation varied with the bending stiffness. The boundary conditions had little influence on the cables with small bending stiffness, and they could be treated as hinged-hinged or fixed-fixed for simple. On the contrary, the boundary conditions had a significant influence on the force estimation for the cables with medium bending stiffness and large bending stiffness, and should be treated as unknown boundary conditions in actual application. 


\section{Abbreviations}

CAE: Considering Angel Effect; EB: Elastic Boundary; EF: Estimated Force; FF: Fundamental Frequency; RCF: Reference cable Force; RE: Relative Error; WCAE: Without Considering Angel Effect

\section{Acknowledgements}

Special thanks to the constructive comments by the anonymous reviewers.

\section{Authors' contributions}

Authors' contributions to this work are shown below: Wen-Yu He: Methodology, Writing-Original Draft. Fan-Cheng Meng: Numerical Analysis. Wei-Xin Ren: Conceptualization, Writing-Review \& Editing. The authors read and approved the final manuscript.

\section{Funding}

The study is partially supported by the National Natural Science Foundation of China (No. 51878234 and 51778204), Fundamental Research Funds for the Central Universities (No. JZ2019HGPA0101), and Shenzhen Science and Technology Program (No. KQTD20180412181337494).

\section{Availability of data and materials}

The data and materials in the current study are available from the corresponding author on reasonable request.

\section{Declarations}

\section{Competing interests}

The authors declare that they have no competing interests.

\section{Author details}

${ }^{1}$ Department of Civil Engineering, Hefei University of Technology, Hefei 230009, Anhui Province, China. ${ }^{2}$ Anhui Engineering Laboratory for Infrastructural Safety Inspection and Monitoring, Hefei 230009, Anhui Province, China. ${ }^{3}$ College of Civil and Transportation Engineering, Shenzhen University, Shenzhen 518061, Guangdong Province, China.

Received: 3 January 2021 Accepted: 18 February 2021

Published online: 06 May 2021

\section{References}

Ceballos MA, Prato CA (2008) Determination of the axial force on stay cables accounting for their bending stiffness and rotational end restraints by free vibration tests. J Sound Vib 317(1-2):127-141

Chen CC, Wu WH, Chen SY, Lai G (2018) A novel tension estimation approach for elastic cables by elimination of complex boundary condition effects employing mode shape functions. Eng Struct 166:152-166

Cunha A, Caetano E, Delgado R (2001) Dynamic tests on large cable-stayed bridge. J Bridge Eng, ASCE 6(1):54-62

Dan DH, Chen YY, Yan XF (2014) Determination of cable force based on the corrected numerical solution of cable vibration frequency equations. Struct Eng Mech 50(1):37-52

Fang Z, Wang JQ (2012) Practical formula for cable tension estimation by vibration method. J Bridge Eng, ASCE 17(1):161164

Feng H, Liu XC, Wu B, Wu DH, Zhang XD, He CF (2019) Temperature-insensitive cable tension monitoring during the construction of a cable-stayed bridge with a custom-developed pulse elasto-magnetic instrument. Struct Health Monit 18(5-6):1982-1994

Huang YH, Fu JY, Wang RH, Gan Q, Liu AR (2015) Unified practical formulas for vibration-based method of tension estimation. Adv Struct Eng 18(3):405-422

Huang YH, Fu JY, Wang RH, Gan Q, Rao R, Liu AR (2014) Practical formula to calculate tension of vertical cable with hingedfixed conditions based on vibration method. J Vibroeng 16(2):997-1009

Irvine HM, Caughey TK (1974) The linear theory of free vibration of a suspended cable. Proc Roy Soc Lond Math Phys Sci 341(1626):299-315

Kim BH, Park TH, Shin HY, Yoon TY (2007) A comparative study of the tension estimation methods for cable supported bridges. Int I Steel Struct 7(1):77-84

Ma L (2017) A highly precise frequency-based method for estimating the tension of an inclined cable with unknown boundary conditions. J Sound Vib 409:56-80

Ma L, Xu H, Munkhbaatar T, Li SF (2021) An accurate frequency-based method for identifying cable tension while considering environmental temperature variation. J Sound Vib 490:115693

Mehrabi AB, Tabatabai H (1998) Unified finite difference formulation for free vibration of cable. J Struct Eng ASCE 124(11): 1313-1322

Ren WX, Chen G, Hu WH (2005) Empirical formulas to estimate cable tension by cable fundamental frequency. Struct Eng Mech 20(3):363-380

Ren WX, Hu WH (2009) Cable modal parameter identification I: theory. J Eng Mech, ASCE 135(1):41-50

Shi X, Zhu S (2018) Nonlinear impact of negative stiffness dampers on stay cables. Struct Monit Maint 5(1):15-38

Tabatabai H, Mehrabi AB, Morgan BJ, Lotfi HR (1997) Nondestructive bridge evaluation technology: bridge stay cable condition assessment. Report to the Federal Highway Administration. Construction Technology Laboratories, Inc., Skokie, IL

Tian YD, Zhang C, Jiang S, Zhang J, Duan WH (2021) Noncontact cable force estimation with unmanned aerial vehicle and computer vision. Comput Aided Civ Inf 36(1):73-88

Yan BF, Chen WB, Yu JY, Jiang XM (2019) Mode shape-aided tension force estimation of cable with arbitrary boundary conditions. J Sound Vib 440:315-331 
Yan BF, Yu JY, Soliman M (2015) Estimation of cable tension force independent of complex boundary conditions. J Eng Mech, ASCE 141(1): 06014015

Zhang X, Peng JY, Cao MS, Damjanovic' D, Ostachowicz W (2020) Identification of instantaneous tension of bridge cables from dynamic responses: strict algorithm and applications. Mech Syst Signal Pr 142:106729

Zhao WJ, Zhang GW, Zhang J (2020) Cable force estimation of a long-span cable-stayed bridge with microwave interferometric radar. Comput Aided Civ Inf 35:1419-1433

Zhao YB, Wang ZQ, Zhang XY, Chen LC (2017) Effects of temperature variation on vibration of a cable-stayed beam. Int J Struct Stab Dy 17(10):1750123

Zui H, Shinke T, Namita Y (1996) Practical formulas for estimation of cable tension by vibration method. J Struct Eng ASCE 122(6):651-656

\section{Publisher's Note}

Springer Nature remains neutral with regard to jurisdictional claims in published maps and institutional affiliations.

Submit your manuscript to a SpringerOpen ${ }^{0}$ journal and benefit from:

- Convenient online submission

- Rigorous peer review

- Open access: articles freely available online

High visibility within the field

Retaining the copyright to your article

Submit your next manuscript at $\boldsymbol{\nabla}$ springeropen.com 